\title{
Determinación de los niveles de procalcitonina para el diagnóstico de sepsis neonatal
}

\author{
Determination of the procalcitonin levels \\ for the diagnosis of neonatal sepsis
}

Carlos Robledo-Restrepo MD', Natalia Maldonado-Lizarazo MSc², Maria C. Noreña-Velásquez MD³, Nathalia Figueroa-Mejía MD³, Juan R. Lopera-Ramírez MD³, Erika Harry-Posada MD³,

Jaime Robledo-Restrepo $\mathrm{PhD}^{4}$

Introducción: la sepsis es la principal causa de morbi-mortalidad en neonatos; sin embargo, debido a la poca especificidad de los signos clínicos en esta población el diagnóstico clínico precoz se considera dificil. Objetivos: evaluar la utilidad de la procalcitonina para el diagnóstico de sepsis en neonatos con diagnóstico presuntivo de esta. Materiales y métodos: se realizó un estudio descriptivo prospectivo en pacientes entre cero y 30 días de nacidos que ingresaron a las Unidades de Cuidados Neonatales de la Clínica El Rosario, sede Centro y sede El Tesoro (Medellín, Colombia), durante septiembre de 2010 y septiembre de 2011, con sospecha de sepsis neonatal o que presentaron un cuadro compatible durante su permanencia. Resultados: de los 65 neonatos captados se confirmó el diagnóstico clínico de sepsis en 39 (60\%). No se encontraron diferencias significativas entre los grupos de neonatos con y sin sepsis respecto a los signos clínicos, parámetros hematológicos o la presencia de alteraciones morfológicas en los eritrocitos. La mediana de los marcadores de infección evaluados, procalcitonina y proteína C reactiva, fueron significativamente superiores en los neonatos con sepsis, así como la proporción de los resultados de procalcitonina y proteína C reactiva interpretados como anormales de acuerdo a los valores de referencia que, en el caso de la procalcitonina, fueron ajustados de acuerdo a las horas transcurridas desde el nacimiento. Conclusiones: los resultados de este estudio demuestran que los reactantes de fase aguda como la procalcitonina y proteína C reactiva son los mejores indicadores de sepsis en neonatos.

Palabras clave: cuidado intensivo neonatal, sepsis, procalcitonina, calcitonina, diagnóstico.

'Médico, especialista en Microbiología y Parasitología Médicas, especialista en Patología clínica. Director Médico, Laboratorio Médico de Referencia, Clínica El Rosario. Medellín, Colombia. Correo electrónico: crobledo@labmedico.co. Teléfono: (057) (4) 2959000 ext. 9033

${ }^{2}$ Microbióloga y Bioanalista, MSc en Epidemiología. Coordinadora de Investigaciones, Laboratorio Médico de Referencia. Medellín, Colombia.

${ }^{3}$ Médico, especialista en Pediatría, Clínica El Rosario. Medellín, Colombia.

${ }^{4}$ Médico, especialista en Microbiología y Parasitología Médicas. PhD en Ciencias Médicas. Subdirector Médico, Laboratorio Médico de Referencia, Clínica El Rosario. Jefe de la Unidad de Bacteriología y Micobacterias, Corporación para Investigaciones Biológicas (CIB). Profesor titular Escuela de Ciencias de la Salud, Universidad Pontificia Bolivariana. Medellín, Colombia.

Conflicto de intereses: los autores declaran que no tienen conflicto de intereses Medicina \& Laboratorio 2015; 21 : 85-92

Módulo 19 (Investigación), número 30. Editora Médica Colombiana S.A. $2015^{\odot}$

Recibido el 28 de enero de 2015; aceptado el 17 de febrero de 2015 
Introduction: sepsis is the leading cause of morbidity and mortality in neonates; however, due to the low specificity of signs and symptoms in this population the early clinical diagnosis is considered difficult. Objectives: to evaluate the usefulness of procalcitonin for the diagnosis of sepsis in neonates with presumptive diagnosis of sepsis. Materials and methods: A descriptive prospective study was made in patients from zero to 30 days of born that were admitted to the Neonatal Care Unit from Clínica El Rosario (Medellin, Colombia), headquarters Centro and headquarters El Tesoro, between September 2010 and September 2011 , with suspicious of neonatal sepsis or that presented a compatible clinical with sepsis during their permanence. Results: From 65 captured neonates, it was confirmed a clinical diagnosis of sepsis in 39 (60\%). No significant differences were observed between neonates with and without sepsis related to clinical and haematological parameters or morphological abnormalities in erythrocytes. The median values of the infection markers evaluated, C-reactive protein and procalcitonin, were significantly higher in neonates with sepsis, as well as the proportion of procalcitonin and C-reactive protein results interpreted as abnormal according to the reference values, that in the case of procalcitonin were adjusted according with the hours since birth. Conclusions: The results of this study demonstrate that acute phase reactants such as C-reactive protein and procalcitonin are the best indicators of neonatal sepsis.

Keywords: neonatal intensive care, sepsis, procalcitonin, calcitonin, diagnosis.

Robledo-Restrepo C, Maldonado-Lizarazo N, Robledo-Restrepo J. Determinación de los niveles de procalcitonina para el diagnóstico de sepsis neonatal. Medicina \& Laboratorio 2015; 21 : 85-92.

L a sepsis es la principal causa de morbi-mortalidad en el período neonatal, que aumenta los costos asociados a los cuidados neonatales para los servicios de salud. Las tasas de mortalidad por sepsis neonatal oscilan entre el $5 \%$ y el $60 \%$, con los valores más altos registrados en los países de bajos ingresos. La Organización Mundial de la Salud (OMS) estima que un millón de muertes al año se deben a la sepsis neonatal y que el $42 \%$ de estas ocurren en la primera semana de vida [I]. Debido a la poca especificidad de los signos clínicos y síntomas de la sepsis en esta población [2], el diagnóstico clínico precoz es difícil, razón por la cual una buena experiencia clínica y la disponibilidad de exámenes complementarios de laboratorio ayudan en el diagnóstico temprano.

El aislamiento de un microorganismo en sangre o un líquido corporal normalmente estéril es el método más específico para establecer el diagnóstico de sepsis neonatal. Otras pruebas de laboratorio como los índices y recuentos leucocitarios, la medición de la proteína C reactiva cuantitativa y de interleuquinas se utilizan en la práctica clínica para diferenciar la sepsis neonatal de otras entidades que pueden cursar con las mismas manifestaciones clínicas [2]. Recientemente, la procalcitonina, producida por los hepatocitos y los macrófagos, ha sido estudiada como un reactante de fase aguda tanto en la población adulta como en la neonatal y pediátrica $[3,4]$

La concentración sérica de la procalcitonina comienza a elevarse cuatro horas después de la exposición a la endotoxina bacteriana, con un pico entre las seis y ocho horas, y permanece elevada durante al menos 24 horas. La vida media es de aproximadamente 25 a 30 horas, y su concentración sérica no se encuentra afectada por la edad gestacional [5]. Además, este marcador ha sido evaluado en los últimos años como una guía para la terapia antibiótica y sus valores han mostrado una correlación con la severidad de la infección, con implicaciones en el pronóstico del paciente [6]. El objetivo de este estudio fue evaluar la utilidad de la determinación de los niveles de procalcitonina en el diagnóstico de sepsis en una población de neonatos con diagnóstico presuntivo de esta entidad. 


\section{Materiales y métodos}

Se realizó un estudio descriptivo prospectivo en las Unidades de Cuidados Neonatales de la Clínica El Rosario sede Centro y sede El Tesoro ubicadas en la ciudad de Medellín, Colombia, entre el 6 de septiembre de 2010 y el 22 de septiembre de 201 I. En el estudio se incluyeron pacientes entre cero y 30 días de nacidos que ingresaron al servicio con sospecha de sepsis neonatal o que durante su estancia hospitalaria presentaron un cuadro compatible con este diagnóstico.

Fueron excluidos del estudio los pacientes que estuvieron sometidos a cirugía dentro de los siete días previos a la sospecha de la sepsis, aquellos que estaban recibiendo terapia antibiótica al momento del diagnóstico o que la hubieran recibido en las 48 horas previas y los pacientes con enfermedades severas concomitantes.

Al momento de la sospecha de la sepsis, y previo al inicio de la terapia antibiótica, a todos los pacientes se les realizó venopunción para la determinación de los niveles de procalcitonina utilizando el sistema VIDAS $^{\circledR}$ (BioMérieux, Marcy l'Etoile, Francia) y de proteína C reactiva mediante el analizador Vitros ${ }^{\circledR} 250$ Chemistry System (Ortho-Clinical Diagnostics, Nueva York, Estados Unidos), realización del hemoleucograma en el equipo COULTER ${ }^{\circledR}$ LH 500 (Beckman Coulter Inc., California, Estados Unidos) y un juego de botellas de hemocultivos aerobios para el sistema BacT/ALERT ${ }^{\circledR}$ (BioMérieux, Marcy l'Etoile, Francia). Se realizó una nueva medición de proteína $C$ reactiva y de procalcitonina entre las 12 y 24 horas después de la primera toma de muestra.

La medición de los niveles de procalcitonina se realizó en muestras de suero por el método de enzimoinmunoensayo (B.R.A.H.M.S. PCT ${ }^{\mathrm{TM}}$ ) con una detección final por fluorescencia en el equipo $\mathrm{VIDAS}^{\circledR}$. Se consideraron los valores de referencia específicos por horas de nacimiento para la interpretación del resultado de la procalcitonina en los pacientes menores de dos días de vida, teniendo en cuenta la elevación fisiológica de la procalcitonina en las primeras 48 horas del nacimiento [7]. A partir del tercer día de vida un valor de procalcitonina mayor que 0,5 ng/ $\mathrm{mL}$ fue considerado como anormal.

Para el diagnóstico definitivo de la presencia de sepsis neonatal, una vez finalizada la recolección de información, se realizó una revisión retrospectiva de las historias clínicas por dos médicos pediatras que confirmaron el diagnóstico clínico de sepsis.

Este estudio contó con la evaluación y aprobación del Comité de Ética e Investigaciones de las instituciones hospitalarias en las que se realizó el estudio. Se solicitó la autorización firmada del responsable de cada menor como criterio necesario para participar en el estudio.

\section{Recolección de datos}

La información fue obtenida de las historias clínicas institucionales de acuerdo a un formulario estructurado que incluyó variables demográficas, características clínicas de los pacientes y los resultados de los exámenes de laboratorio realizados como parte del diagnóstico de sepsis.

\section{Análisis estadístico}

Se realizaron análisis de frecuencias y medidas de tendencia central de las variables en cada uno de los grupos de estudio de acuerdo a la clasificación final de sepsis utilizando el programa 
estadístico SPSS 15,0 (SPSS ${ }^{\circledR}$ Inc., Illinois, Estados Unidos). Se emplearon las pruebas de $t$ de Student y de $U$ de Mann-Whitney para determinar las diferencias entre los grupos con respecto a las variables cuantitativas de acuerdo a la distribución de las mismas. Para las variables categóricas se empleó la prueba de $\mathrm{Chi}^{2}$ o la prueba exacta de Fisher. Un valor p menor o igual que 0,05 se consideró como estadísticamente significativo.

\section{Resultados}

En total se incluyeron en el estudio 65 pacientes, 40 (61,5\%) de ellos de sexo masculino. La edad gestacional fue en promedio de 35,7 semanas (rango: 24 a 40 semanas) y la media de la edad de los pacientes de 5,2 días ( 0 a 46 días). El peso promedio fue de 2.528 gramos y una talla promedio de $45,6 \mathrm{~cm}$. Un total de 33 (50,8\%) pacientes fueron nacidos pretérmino y los signos clínicos más frecuentemente encontrados fueron dificultad respiratoria $(61,5 \%)$, ictericia $(36,9 \%)$, cianosis $(29,2 \%)$ y vómito $(23,1 \%)$ (véase tabla I).

En la tabla 2 se describen los hallazgos anormales en los parámetros hematológicos de los pacientes incluidos en el estudio de acuerdo a los valores de referencia para esta población vigentes en el laboratorio. Un total de 22 (33,8\%) pacientes presentaron recuentos anormales de leucocitos y 53 (81,5\%) un porcentaje alterado de neutrófilos. En 42 (64,6\%) pacientes se observó policromatofilia, en $40(61,5 \%)$ la presencia de bandas y en $15(23,1 \%)$ la presencia de eritroblastos.

Entre la primera y la segunda toma de muestra para el análisis de la proteína $C$ reactiva transcurrieron en promedio 19,91 horas y para la procalcitonina 17,68 horas. La mediana del valor de proteína $C$ reactiva y procalcitonina en la primera toma de muestra fue de I,05 mg/dL y $3,40 \mathrm{ng} / \mathrm{mL}$, respectivamente, entretanto en la segunda toma de muestra estos valores fueron de 1,2 mg/dL y 2,78 $\mathrm{ng} / \mathrm{mL}$, respectivamente. Los resultados de cada paciente se interpretaron de acuerdo a los intervalos biológicos de referencia para ambos analitos, considerando un valor anormal de proteína $C$ reactiva mayor que I mg/dL, mientras que para la procalcitonina se tuvo en cuenta la elevación fisiológica de este analito en las primeras 48 horas de vida de acuerdo a lo descrito por Chiesa y colaboradores [7], y en los pacientes con más de 48 horas de nacidos un valor anormal mayor que $0,5 \mathrm{ng} / \mathrm{mL}$. 


\begin{tabular}{|c|c|c|c|}
\hline Variable & Valor de referencia & $\mathrm{n}$ & Porcentaje (\%) \\
\hline Recuento anormal de leucocitos $\left(10^{3} / \mu \mathrm{L}\right)$ & $4-11$ & 22 & 33,8 \\
\hline Recuento anormal de neutrófilos (\%) & $40-65$ & 53 & 81,5 \\
\hline Recuento anormal de plaquetas $\left(10^{3} / \mu \mathrm{L}\right)$ & $140-440$ & $\mid 1$ & 16,9 \\
\hline Recuento anormal de eritrocitos $\left(10^{6} / \mu \mathrm{L}\right)$ & $3,8-5,2$ & 16 & 24,6 \\
\hline Valores anormales de hemoglobina ( $\mathrm{g} / \mathrm{dL}$ ) & $12-16$ & 16 & 24,6 \\
\hline Valores anormales de hematocrito (\%) & $35-47$ & 23 & 35,4 \\
\hline Presencia de policromatofilia & - & 42 & 64,6 \\
\hline Presencia de microcitos & - & 9 & 13,8 \\
\hline Presencia de macrocitos & - & 12 & 18,5 \\
\hline Presencia de eritroblastos & - & 15 & 23,1 \\
\hline Presencia de hipocromía & - & 3 & 4,6 \\
\hline Presencia de acantocitos & - & 2 & 3,1 \\
\hline Presencia de bandas & - & 40 & 61,5 \\
\hline
\end{tabular}

La proporción de pacientes con resultado anormal en ambas pruebas en las dos tomas de muestra, así como los resultados de los hemocultivos se describen en la tabla 3. La revisión retrospectiva de las historias clínicas por dos médicos pediatras confirmó el diagnóstico clínico de sepsis en 39 (60,0\%) pacientes y en los 26 restantes no se confirmó la presencia de la sepsis neonatal. En la tabla 4 se reportan las características clínicas del grupo con diagnóstico clínico de sepsis y del grupo sin sepsis neonatal. No se encontraron diferencias significativas entre los grupos con respecto a las variables clínicas y demográficas, con excepción del peso promedio en gramos $(p=0,049)$, el cual fue mayor en el grupo de neonatos con sepsis.

\begin{tabular}{|c|c|c|}
\hline Variable & $\mathrm{n}$ & Porcentaje (\%) \\
\hline Resultado anormal de proteína $C$ reactiva en la primera muestra $(n=63)$ & 32 & 50,8 \\
\hline $\begin{array}{l}\text { Resultado anormal de procalcitonina en la primera muestra ajustado por horas } \\
\text { de nacimiento }(n=65)\end{array}$ & 27 & 41,5 \\
\hline Resultado anormal de proteína $C$ reactiva en la segunda muestra $(n=61)$ & 35 & 57,4 \\
\hline $\begin{array}{l}\text { Resultado anormal procalcitonina en la segunda muestra ajustado por horas } \\
\text { de nacimiento }(n=60)\end{array}$ & 27 & 45,0 \\
\hline Hemocultivos positivos $(n=64)$ & 8 & 12,5 \\
\hline
\end{tabular}

No se encontraron diferencias importantes entre el grupo con sepsis y el grupo sin sepsis con respecto a los parámetros hematológicos ni las anormalidades morfológicas de los eritrocitos (véase tabla 5); sin embargo, los valores de la mediana de los marcadores de infección fueron significativamente superiores en los neonatos con diagnóstico clínico de sepsis, así como la proporción de los resultados de proteína $C$ reactiva y procalcitonina interpretados como anormales de acuerdo a los valores de referencia, en el caso de la última, ajustando la interpretación de acuerdo a las horas transcurridas desde el nacimiento (véase tabla 6). En un paciente no fue posible obtener muestra para realizar el hemocultivo, cuatro neonatos del grupo de pacientes con sepsis tuvieron hemocul- 
tivo positivo (10,3\%) y el mismo número de pacientes en el grupo sin sepsis (16,0\%), lo que no representa una diferencia estadísticamente significativa $(p=0,70 \mathrm{I})$.

Del grupo de pacientes con sepsis con hemocultivos positivos dos tuvieron aislamientos de Staphylococcus epidermidis en una de las dos botellas, en uno se aisló Staphylococcus hominis en las dos botellas y en el otro se obtuvo Kocurea rosea en ambas botellas. Tres de los cuatro pacientes con hemocultivos positivos en el grupo sin sepsis tuvieron un aislamiento de Staphylococcus epidermidis y en el otro se aisló Candida parapsilosis; en los cuatro casos todos los aislamientos fueron obtenidos sólo en una de las dos botellas de los hemocultivos tomados.

\section{Discusión}

La sepsis es una entidad de alto impacto en la población neonatal. El diagnóstico temprano de esta entidad es difícil debido a que los signos clínicos iniciales son inespecíficos y similares a otros procesos de etiología no infecciosa [7]. En el presente estudio, que incluyó 65 neonatos con sospecha de sepsis, en los que la presencia de signos clínicos fue similar en el grupo de pacientes con confirmación de sepsis y sin sepsis, se ratifica la falta de especificidad de las características clínicas y, por ende, la dificultad de un diagnóstico clínico temprano.

El aislamiento de las bacterias presentes en la sangre por medio del cultivo se considera el estándar de oro para el diagnóstico de sepsis

\begin{tabular}{|c|c|c|c|}
\hline \multirow[b]{2}{*}{ Variable } & \multicolumn{3}{|c|}{ N. ${ }^{\circ}(\%)$ de pacientes } \\
\hline & $\begin{array}{l}\text { Con sepsis } \\
(n=39)\end{array}$ & $\begin{array}{l}\text { Sin sepsis } \\
(n=26)\end{array}$ & valor $p$ \\
\hline Sexo masculino & $25(64,1)$ & I5 $(57,7)$ & 0,603 \\
\hline $\begin{array}{l}\text { Recién nacido } \\
\text { pretérmino }\end{array}$ & $18(46,2)$ & I $5(57,7)$ & 0,417 \\
\hline $\begin{array}{l}\text { Edad gestacional } \\
\text { (semanas) }\end{array}$ & 35,97 & 35,38 & 0,207 \\
\hline Edad (días) & 6,45 & 3,42 & 0,761 \\
\hline Peso (gramos) & 2,657 & 2,340 & 0,049 \\
\hline Talla (cm) & 46,2 & 44,7 & 0,076 \\
\hline Hipotermia & $3(7,7)$ & $0(0,0)$ & 0,269 \\
\hline Hipertermia & $4(10,3)$ & $14(53,8)$ & 0,144 \\
\hline Dificultad respiratoria & $26(66,7)$ & $14(53,8)$ & 0,298 \\
\hline Apnea & $5(12,8)$ & $4(15,4)$ & 1,000 \\
\hline Cianosis & $12(30,8)$ & $7(26,9)$ & 0,738 \\
\hline Tos & $2(5,1)$ & $0(0,0)$ & 0,513 \\
\hline Taquicardia & $7(\mid 7,9)$ & I $(3,8)$ & 0,131 \\
\hline Bradicardia & I $(2,6)$ & $2(7,7)$ & 0,559 \\
\hline Hipotensión & $4(10,3)$ & $2(7,7)$ & 1,000 \\
\hline Perfusión pobre & $4(10,3)$ & $2(7,7)$ & 1,000 \\
\hline Anorexia & $4(10,3)$ & I $(3,8)$ & 0,640 \\
\hline Succión pobre & $8(20,5)$ & $6(23,1)$ & 0,805 \\
\hline Dolor a la palpación & $3(7,7)$ & $\mathrm{I}(3,8)$ & 0,644 \\
\hline Diarrea & I $(2,6)$ & $0(0,0)$ & 1,000 \\
\hline Vómito & $10(25,6)$ & $5(19,2)$ & 0,548 \\
\hline Enterorragia & $0(0,0)$ & $I(3,8)$ & 0,400 \\
\hline Distensión abdominal & $3(7,7)$ & $5(19,2)$ & 0,250 \\
\hline Irritabilidad & $8(20,5)$ & $3(11,5)$ & 0,503 \\
\hline Convulsiones & $2(5,1)$ & $0(0,0)$ & 0,513 \\
\hline Fontanela tensa & I $(2,6)$ & $0(0,0)$ & 1,000 \\
\hline Decaimiento & $6(15,4)$ & $3(11,5)$ & 0,731 \\
\hline Petequias & $0(0,0)$ & $I(3,8)$ & 1,000 \\
\hline Ictericia & $14(35,9)$ & $10(38,5)$ & 0,834 \\
\hline Anemia & $4(10,3)$ & $I(3,8)$ & 0,640 \\
\hline
\end{tabular}

[8]; sin embargo, esta prueba tiene algunas limitaciones para proveer un diagnóstico oportuno y certero, debido a que los resultados pueden tomar entre 24 y 48 horas y requiere la inoculación de 0,5 a I,0 mL de sangre, que considerando que el 60\% al 70\% de los niños tienen un bajo nivel de bacteriemia, compromete la sensibilidad de la prueba; además, la carga bacteriana puede ser baja cuando las madres han recibido antibióticos antes o durante el parto. Una dificultad importante que compromete la utilidad de los hemocultivos son las altas tasas de contaminación asociadas a los problemas técnicos de una punción venosa estéril en esta población. Esto trae como consecuencia la dificultad de interpretar el aislamiento de estafilococos coagulasa negativos en los hemoculti- 
vos, ya que estos organismos hacen parte de la microbiota normal de la piel, pero pueden ser patógenos en los prematuros y recién nacidos con catéteres vasculares $[9,10]$. Dadas estas dificultades, se requiere un marcador diagnóstico que sea útil para decidir el inicio de la terapia antimicrobiana de manera oportuna y que, a su vez, permita hacer seguimiento y suspender la administración innecesaria de antibióticos.

En el presente estudio los reactantes de fase aguda como la proteína $C$ reactiva y la procalcitonina fueron los mejores indicadores de sepsis neonatal en la población estudiada, pues los valores promedio fueron significativamente más altos en el grupo de neonatos con diagnóstico definitivo de sepsis clínica respecto a los confirmados sin sepsis. Esto ha sido reportado previamente en varios estudios en los que se ha demostrado que tanto los niveles de proteína $C$ reactiva como de procalcitonina se incrementan apreciablemente durante el curso de una infección bacteriana sistémica, lo que ha permitido evaluarlos como marcadores para la detección precoz de la sepsis tanto de aparición temprana como la sepsis nosocomial $[11,12]$. Algunos estudios han demostrado un

\begin{tabular}{|c|c|c|c|}
\hline \multirow[b]{2}{*}{ Variable } & \multicolumn{3}{|c|}{ N. ${ }^{\circ}(\%)$ de pacientes } \\
\hline & $\begin{array}{l}\text { Con sepsis } \\
(n=39)\end{array}$ & $\begin{array}{l}\text { Sin sepsis } \\
(n=26)\end{array}$ & Valor $p$ \\
\hline $\begin{array}{l}\text { Recuento anormal } \\
\text { de leucocitos }\end{array}$ & $15(38,5)$ & $7(26,9)$ & 0,335 \\
\hline $\begin{array}{l}\text { Recuento anormal } \\
\text { de neutrófilos }\end{array}$ & $32(82,1)$ & $21(80,8)$ & 1,000 \\
\hline $\begin{array}{l}\text { Recuento anormal } \\
\text { de plaquetas }\end{array}$ & $26(66,7)$ & $14(53,8)$ & 0,237 \\
\hline $\begin{array}{l}\text { Recuento anormal } \\
\text { de eritrocitos }\end{array}$ & $12(30,8)$ & $4(15,4)$ & 0,158 \\
\hline $\begin{array}{l}\text { Presencia de } \\
\text { policromatofilia }\end{array}$ & $28(7 \mid, 8)$ & $14(53,8)$ & 0,101 \\
\hline $\begin{array}{l}\text { Presencia de } \\
\text { microcitos }\end{array}$ & $8(20,5)$ & $\mathrm{I}(3,8)$ & $0,07 \mid$ \\
\hline $\begin{array}{l}\text { Presencia de } \\
\text { macrocitos }\end{array}$ & $10(25,6)$ & $2(7,7)$ & 0,101 \\
\hline $\begin{array}{l}\text { Presencia de } \\
\text { eritroblastos }\end{array}$ & $7(17,9)$ & $8(30,8)$ & 0,252 \\
\hline $\begin{array}{l}\text { Presencia de } \\
\text { hipocromía }\end{array}$ & $3(7,7)$ & $0(0)$ & 0,265 \\
\hline $\begin{array}{l}\text { Presencia de } \\
\text { acantocitos }\end{array}$ & $2(5,1)$ & $0(0)$ & 0,510 \\
\hline $\begin{array}{l}\text { Presencia de } \\
\text { bandas }\end{array}$ & $26(66,7)$ & $14(53,8)$ & 0,237 \\
\hline $\begin{array}{l}\text { Valores anormales } \\
\text { de hemoglobina }\end{array}$ & $13(33,3)$ & $3(11,5)$ & 0,046 \\
\hline $\begin{array}{l}\text { Valores anormales } \\
\text { de hematocrito }\end{array}$ & $18(46,2)$ & $5(19,2)$ & 0,026 \\
\hline
\end{tabular}

\begin{tabular}{|c|c|c|c|c|c|}
\hline \multirow{2}{*}{ Variables } & \multicolumn{2}{|c|}{ Con sepsis $(n=39)$} & \multicolumn{2}{|c|}{ Sin sepsis $(n=26)$} & \multirow{2}{*}{$\begin{array}{l}\text { Valor } \\
\text { P }\end{array}$} \\
\hline & $\mathrm{n}$ & N. ${ }^{\circ}(\%)$ & $\mathrm{n}$ & $N .^{\circ}(\%)$ & \\
\hline $\begin{array}{l}\text { Resultado anormal de la proteína C reactiva en la } \\
\text { toma I }\end{array}$ & 38 & $24(63,2)$ & 25 & $8(32,0)$ & 0,016 \\
\hline $\begin{array}{l}\text { Resultado anormal procalcitonina ajustado por } \\
\text { horas de nacimiento en la toma I }\end{array}$ & 39 & $23(59,0)$ & 26 & $4(15,4)$ & 0,000 \\
\hline $\begin{array}{l}\text { Resultado anormal de la proteína C reactiva en la } \\
\text { toma } 2\end{array}$ & 37 & $28(75,7)$ & 24 & $7(29,2)$ & 0,000 \\
\hline $\begin{array}{l}\text { Resultado anormal procalcitonina ajustado por } \\
\text { horas de nacimiento en la toma } 2\end{array}$ & 38 & $23(60,5)$ & 22 & $4(18,2)$ & 0,001 \\
\hline Hemocultivos positivos & 39 & $4(10,3)$ & 25 & $4(16,0)$ & 0,701 \\
\hline Variables & $n$ & Valor promedio & $n$ & Valor promedio & Valor $\mathrm{p}$ \\
\hline Proteína C reactiva en la toma I (mg/dL) & 38 & 1,30 & 25 & 0,40 & 0,020 \\
\hline Procalcitonina en la toma I ( $\mathrm{ng} / \mathrm{mL})$ & 39 & 4,52 & 26 & 1,78 & 0,010 \\
\hline Proteína C reactiva en la toma 2 (mg/dL) & 37 & 1,50 & 24 & 0,50 & 0,000 \\
\hline Procalcitonina en la toma 2 (ng/mL) & 38 & 5,12 & 22 & 2,44 & 0,044 \\
\hline
\end{tabular}

Nota: por la dificultad que existe para la venopunción en este grupo de edad algunos de los pacientes no contaron con todas las mediciones establecidas para este estudio 
desempeño superior de la procalcitonina en comparación de la proteína $C$ reactiva en términos del diagnóstico temprano de la sepsis neonatal $[13, \mid 4]$.

Un metanálisis realizado por Vouloumanou y colaboradores (201 I), que incluyó 16 estudios que involucraban un total de 1.959 neonatos, demostró que la sensibilidad agrupada de la procalcitonina para el diagnóstico de sepsis neonatal era de $81 \%$ y la especificidad de $79 \%$; sin embargo, los autores mencionan que aunque la determinación de la procalcitonina en el momento de la presentación clínica de la sepsis tiene muy buena precisión diagnóstica para discriminar entre los recién nacidos con sepsis y aquellos con otras condiciones, al igual que la proteína C-reactiva y el recuento total de leucocitos, no puede identificar por sí misma correctamente al 100\% de los neonatos sépticos [4].

Una importante limitación del presente estudio es el pequeño número de neonatos estudiados, razón por la que se recomienda la realización de futuras investigaciones con un número mayor de sujetos que permitan confirmar el papel de la determinación de la procalcitonina en el diagnóstico de la sepsis neonatal.

\section{Conclusiones}

En resumen, la procalcitonina demostró ser un buen marcador que contribuye al diagnóstico inicial de la sepsis neonatal, aunque no fue capaz de identificar todos los neonatos con sepsis. El uso concomitante de otros marcadores y la valoración clínica, aunque menos sensibles, pueden en conjunto aumentar el valor predictivo de la procalcitonina.

\section{Bibliografía}

I. Edmond K, Zaidi A. New approaches to preventing, diagnosing, and treating neonatal sepsis. PLoS Med 2010; 7: el000213.

2. Camacho-Gonzalez A, Spearman PW, Stoll BJ. Neonatal infectious diseases: evaluation of neonatal sepsis. Pediatr Clin North Am 2013; 60: 367-389.

3. Wacker C, Prkno A, Brunkhorst FM, Schlattmann P. Procalcitonin as a diagnostic marker for sepsis: a systematic review and meta-analysis. Lancet Infect Dis 2013; 13: 426435.

4. Vouloumanou EK, Plessa E, Karageorgopoulos DE, Mantadakis E, Falagas ME. Serum procalcitonin as a diagnostic marker for neonatal sepsis: a systematic review and metaanalysis. Intensive Care Med 201 1; 37: 747-762.

5. Shah BA, Padbury JF. Neonatal sepsis: an old problem with new insights. Virulence 2014; 5: 170-178.

6. Lee $\mathrm{H}$. Procalcitonin as a biomarker of infectious diseases. Korean J Intern Med 2013; 28: 285-291.

7. Chiesa C, Panero A, Rossi N, Stegagno M, De Giusti M, Osborn JF, et al. Reliability of procalcitonin concentrations for the diagnosis of sepsis in critically ill neonates. Clin Infect Dis 1998; 26: 664-672.

8. Marchant EA, Boyce GK, Sadarangani M, Lavoie PM. Neonatal sepsis due to coagulase-negative staphylococci.
Clin Dev Immunol 2013; 2013: 586076.

9. Resch B, Hofer N, Müller W. Challenges in the Diagnosis of Sepsis of the Neonate. En: Azevedo L, ed. Sepsis - An Ongoing and Significant Challenge. Rijeka, Croacia: InTech; 2012: 233-248

10. Paolucci M, Landini MP, Sambri V. How can the microbiologist help in diagnosing neonatal sepsis? Int J Pediatr 2012; 2012: 120139 .

II. Simonsen KA, Anderson-Berry AL, Delair SF, Davies HD. Early-onset neonatal sepsis. Clin Microbiol Rev 2014 27: $21-47$.

12. Pérez Solís D, López Sastre JB, Coto Cotallo GD, Diéguez Junquera MA, Deschamps Mosquera EM, Crespo Hernández M. Procalcitonina para el diagnóstico de sepsis neonatal de origen nosocomial. Anales de Pediatría 2006; 64: 349-353.

13. Sucilathangam G, Amuthavalli K, Velvizhi G, Ashihabegum MA, Jeyamurugan T, Palaniappan N. Early Diagnostic Markers for Neonatal Sepsis: Comparing Procalcitonin (PCT) and C-Reactive Protein (CRP). J Clin Diagn Res 2012; 6: 627-631.

14. Ali AM, Elkhatib WF, Abdelaziz SS. Procalcitonin versus C-Reactive Protein in Neonatal Sepsis. J Immunol Infect Dis 2014; 1: 103-107. 